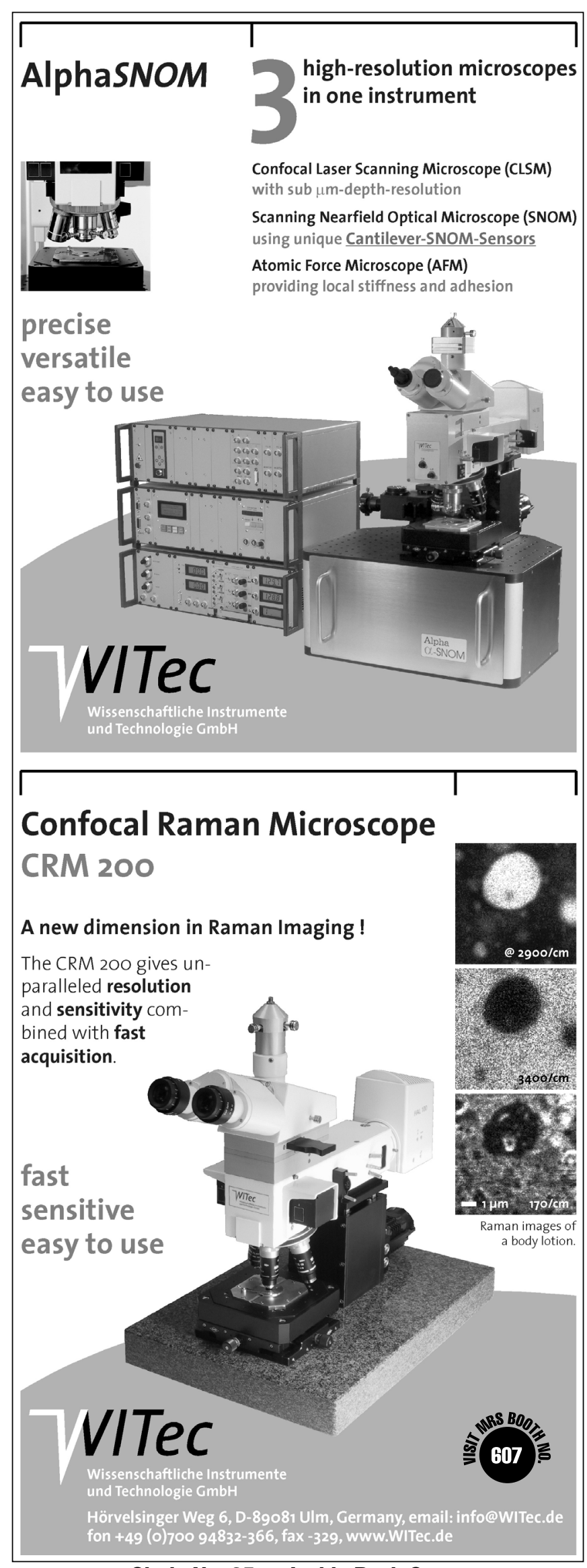

The results demonstrate that high-quality superconducting structures can be realized on a length scale $<100 \mathrm{~nm}$ in in situ fabricated $\mathrm{MgB}_{2}$ films. Furthermore, the results show that the chemical reactivity and volatility of magnesium do not limit FIB processing of structures on the nanoscale level. $\mathrm{MgB}_{2}$ ring structures incorporating nanobridges display Josephson quantum interference effects, which form the basis for the creation of an all-MgB $\mathrm{MQUID}_{2}$. According to the researchers, "This result is an essential step toward sensors and electronic circuits based on this novel superconductor."

SHIMING Wu

\section{Antimicrobial Materials for Medical Implants Prepared Using Sol-Gel Techniques}

Researchers in the Department of Chemistry at the University of North Carolina-Chapel Hill have synthesized stable and tunable NO-releasing siloxane polymer films for use as potential medical implant coatings. The coatings were designed and characterized by Mark Schoenfisch and graduate students Brian Nablo and Ta-Yung Chen. The researchers prepared NO-releasing sol-gel materials by combining various amounts of isobutyltrimethoxysilane (iBuTMOS) and (3-trimethoxysilylpropyl) diethylene-triamine (DET3), casting thin films of the mixture onto glass slides, and then exposing the slides to high pressures of NO. The researchers demonstrated that these materials controllably released low levels of $\mathrm{NO}$, a potent antibacterial agent, and prevented the adhesion of Pseudomonas aeruginosa bacteria. According to Schoenfisch, "Localized NO release may prove to be an effective strategy for lessening the frequency of biofilm formation and implant-related infections." P. aeruginosa was selected for the study because it is a well-characterized, medically relevant bacterium known to actively form biofilms.

As reported in the October 3 issue of the Journal of the American Chemical Society, the NO-releasing materials were prepared by the addition of three mole equivalents of water to a mixture of iBuTMOS and DET3. The mixture was then stirred for $5 \mathrm{~min}$ before casting onto glass slides. The sol-gel-coated slides were allowed to gel, dry, and age for 5-7 days under ambient conditions. The amine group moieties were then converted to diazeniumdiolates (NO donors) by exposure to $5 \mathrm{~atm}$ of $\mathrm{NO}$ for $72 \mathrm{~h}$. NO chemiluminescence studies showed that the gels released NO continuously for up to $24 \mathrm{~h}$. The amount of NO released was tuned by adjusting the fraction of DET3 in the sol.

To test the bacterial adhesion resistance of the NO-releasing coatings, the sol-gel-modified glass slides were exposed to $P$. aeruginosa for $30 \mathrm{~min}$ followed by fixing and staining the adhered cells. Opacity measurements of the slides revealed that the number of adhered cells was reduced by a factor of 2 for $15 \%$ DET3 gels and by a factor of 4 for $45 \%$ DET3 gels compared to control slides without NO-release capabilities. In addition, reduced cell adhesion was not observed on control slides exposed to solutions of $\mathrm{NO}_{2}{ }^{-}$, demonstrating that the $\mathrm{NO}$, and not nitrite, $\mathrm{NO}^{\prime} \mathrm{s}$ major oxidation product, is responsible for the reduced cell adhesion.

To test the stability of the films, the Si content from phosphatebuffered saline soak solutions was measured as a function of solgel immersion time. The Si concentrations in the soak solutions were extremely low, indicating minimal loss of Si from the aminasilane precursors which are covalently linked to the polymer backbone and thereby indicating satisfactory material stability.

These studies show that the NO-releasing materials could inhibit or even prevent bacterial growth on medical implants. Schoenfisch said that complete inhibition of cell adhesion was not achieved because this study was performed at relatively low NO-release rates and high concentrations of cells. His group is currently investigating the effectiveness of the films to reduce the adhesion of other medically relevant bacterial species.

GREGORY KHITROV

Circle No. 35 on Inside Back Cover 